\title{
TWO ALTERNATIVE METHODS FOR ANAERO- BIC CULTIVATION OF BACTEROIDES FRAGILIS ATCC 25285 COMPARED TO THE EFFICIENCY OF CULTIVATION IN A GAS PACK SYSTEM
}

\author{
Neli M. Ermenlieva ${ }^{1}$, Emilia P. Georgieva ${ }^{2}$, Sylvia Y. Stamova ${ }^{3}$, Svetlana F. \\ Georgieva $^{3}$, Silvia G. Mihaylova ${ }^{4}$ \\ 1) Department of Microbiology and Virology, Faculty of Medicine, Medical Uni- \\ versity - Varna, Bulgaria \\ 2) TRS Medical laboratory assistant, Medical College, Medical University- \\ Varna, Bulgaria \\ 3) Department of Pharmaceutical Chemistry, Faculty of Pharmacy, Medical \\ University - Varna, Bulgaria \\ 4) TRS Assistant Pharmacist, Medical College, Medical University of Varna, \\ Bulgaria.
}

\begin{abstract}
Cultivation of anaerobes is not a routine procedure. In most cases, it is carried out in reference laboratories with the help of special equipment, including an anaerobic chamber or anaerobic jar with Gas pack system. There are some older methods so-called the Candle jar system and the Fortner principle.

The aim of the study is to compare two alternative methods for anaerobic cultivation of Bacteroides fragilis ATCC 25285 with cultivation by Gas Pack system, which is a widely used and well studied method.

We made anaerobic cultivation using the Gas Pack system, Candle jar and Fortner principle. The bacteria were seed in two different types of agar media - suitable for anaerobic cultivation Wilkins-Chalgren agar and MuellerHinton agar, useful for antibiotic sensitivity testing.

The results of our study showed that in all bacterial cultures, B. fragilis ATCC 25285 demonstrated heavy growth. The Candle jar system and Fortner principle were found to be a sensitive and cost-effective alternative that might be used in resource-limited settings.
\end{abstract}

Keywords: B. fragilis, cultivation, Fortner principle, Candle jar, alternative methods,

\section{INTRODUCTION}

Bacteroides fragilis are Gram-negative rod-shaped bacteria. Most healthy adult microbiota are dominated by just two bacterial phyla - the Gram-positive Firmicutes (many genera) and the Gram-negative Bacteroidetes (primarily Bacteroides, Alistipes, Parabacteroides, and Prevotella) [1]. At the same time, Bacteroides are a common cause of endogenous infections [2] and the commonest anaerobic bacteria associated with clinical infections, particularly those associated with infections of the mucous membranes and adjacent tissues [3,4]. Bacteroides are obligate anaerobes, but they are one of the most aerotolerant anaerobes, and according to Baughn and Malamy, 2004 [5], they may actually slowly divide in the presence of nanomolar concentrations of oxygen.

Cultivation of anaerobes is not a routine procedure. In most cases, it is carried out in reference laboratories with the help of special equipment, including an anaerobic chamber. More accessible methods include the use of an anaerobic jar in which a Gas pack system is placed to create an anaerobic environment chemically. Other similar methods are the use of vented anaerobe jars (usually consisting of $\mathrm{N}_{2} 85 \%, \mathrm{CO}_{2} 10 \%$ and $\mathrm{H}_{2} 5 \%$ ), anaerobic gassing cannula (supply of stream of anaerobic gas into the culture vessel) [6] and others.

Some older methods of so-called physical and biological anaerobic cultivation are described in the literature. The biological method, which is called the Fortner principle, utilized the ability of rapidly growing facultative anaerobes to reduce the oxygen tension in a closed system, thus making possible the growth of oxygen-sensitive organisms [7, 8] such as Bacteroides. The principle includes using an aerobic organism cultivated together with testing anaerobe into one nutrient medium. The aerobic organism multiplies for the first 24 hours using oxygen in the Petri dish. Then the anaerobic bacteria start to grow, and within the next 24 to 48 hours, we may see the macroscopic visible bacterial colonies.

Anaerobic cultivation by physical method includes using a Candle jar. With the help of a lighted candle in the apparatus creates anaerobic conditions, in the combustion process releases carbon dioxide and consumes oxygen. By combustion of a white wax candle in the candle jar system, 4-5 per cent $\mathrm{CO}_{2}$ is generated with instantaneous lowering of the oxygen level inside the sealed jar, leaving approximately 1-2 per cent un-burnt oxygen $[9,10]$. This technique is not so typical for the purpose 
of anaerobic cultivation; it's more often used for microaerophiles cultivation, which requires lower levels of oxygen than are present in the atmosphere and an elevated concentration of carbon dioxide. The atmosphere most commonly used for culturing Bacteroides contains 5 or $10 \% \mathrm{CO}_{2}$ [6]. At the same time, carbon monoxide can be inhibitory to some anaerobic organisms. However, we decided to investigate Bacteroides fragilis to grow successfully in the Candle jar system.

\section{Aim}

The aim of the study is to compare the effectiveness of three different methods for anaerobic cultivation of Bacteroides fragilis ATCC 25285.

\section{MATERIALS AND METHODS}

The study was conducted in a training microbiological laboratory in Medical university-Varna, Bulgaria, in March 2021. We investigated the possibility of culturing B. fragilis ATCC 25285, creating anaerobic conditions by three different methods.

Anaerobic cultivation using a Gas Pack system. We performed dense seeds of $B$. fragilis in two different types of agar media - suitable for anaerobic cultivation WilkinsChalgren agar and Mueller-Hinton agar, useful for antibiotic sensitivity testing. For the purposes of anaerobic cultivation, we used an anaerobic jar (Schuett-biotec "eco", Germany) with an Anaerob Pack system $\left(\mathrm{H}_{2}+\mathrm{CO}_{2}\right)$ and an indicator with methylene blue for an anaerobic environment. We put the Petri dishes with nutrient media upside down and cultivate them in the incubator for 48 hours at $35^{\circ} \mathrm{C}$. The methodology is performed in triplicate.

Anaerobic cultivation using Candle jar. We made dense seeds of $B$. fragiles on Wilkins-Chalgren agar and Mueller-Hinton agar. The glassware was left with a freely moving lid. The procedure was made in triplicate. We put the Petri dishes upside down in a candle jar, and then we placed a lighted candle on them and closed the lid by carefully sliding it on top. After a minute, the candle flame went out. We sealed the peripheral edges of the vessel with melted paraffin and cultivated bacterial cultures at $35^{\circ} \mathrm{C} / 48$ hours.

Biological method for anaerobic cultivation Fortner principle. We used Wilkins-Chalgren agar and Mueller-Hinton agar for Bacteroides cultivation. With a sterile knife, we cut a strip from the agar, which divided it into two halves. A strain of Bacteroides fragilis was streaked onto one half of the medium, and a strain of Staphylococcus aureus was streaked onto the other half. Then we turned upside down only the lid of the Petri dish and sealed it together with the bottom with melted paraffin. In this way, we closed the glassware hermetically and stopped the supply of oxygen from the outside. The bacteria cultures were incubated at $35^{\circ} \mathrm{C}$ for 48 hours. We repeated the experiment, but $S$. aureus was replaced by a strain of Escherichia coli. All the procedures were performed in triplicate.

\section{RESULTS AND DISCUSSION}

The results of our study showed that in all twentyfour bacterial cultures we performed, B. fragilis demonstrated heavy growth. These 24 bacterial seeds were made following three methods for anaerobic cultivation of Bacteroides fragilis ATCC 25285: cultivation onto Wilkins-Chalgren agar and Mueller-Hinton agar, using Gas Pack system (6 bacterial seeds); cultivation onto Wilkins-Chalgren agar and Mueller-Hinton agar, using a candle jar (6 bacterial seeds); cultivation onto WilkinsChalgren agar and Mueller-Hinton agar, using Fortner principle with strains of Staphylococcus aureus and Escherichia coli (12 bacterial seeds).

Each of the three methods used for anaerobic cultivation of $B$. fragilis proved to be successful. The degree of growth and colony size were similar in all three systems. Interestingly, in three of the six of the WilkinsChalgren agar plates, cultivated by the Fortner principle, the facultative anaerobe microorganisms $E$. coli and $S$. aureus were not successfully cultured. The reason is probably that this type of nutrient medium is intended for strict anaerobes. However, the target anaerobic microorganism had multiplied actively (Figure 1). This confirms the statement of Baughn and Malamy, 2004 [5] that genus Bacteroides are among the most aerotolerant anaerobes and, at certain oxygen concentrations, can survive and multiply. Sealing the plates with paraffin proved to be sufficient to create the necessary conditions for the survival of the strain.

It is necessary to specify that although Bacteroides fragilis ATCC 25285 showed heavy growths cultivated in a Candle jar system, this does not absolutely prove that this technique is suitable for all strict anaerobes. As discussed above, this method does not achieve complete anaerobiosis, and it is possible that the known aerotolerance of Bacteroides makes it suitable for this type of cultivation, unlike other anaerobes. Saha et al., 2016 [10] also share the opinion that the Candle jar is an alternative technique that can be used to reduce the major bulk of oxygen rapidly. However, they present a study in which they use a modified candle-jar system, acidified coppercoated steel wool is used for removal of residual oxygen after candle combustion.

\section{CONCLUSION}

Anaerobic cultivation of bacteria by chemical agents such as the Gas Pack system is a widely used method, and its high efficiency is well studied and known. However, achieving an anaerobic environment by Candle jar and Fortner principle are two alternative methods that are not being used or are being used less frequently or almost not at all. In fact, our study shows that these two methods effectively and with sufficient duration create conditions for the cultivation of strict anaerobe as Bacteroides fragilis. The Candle jar system and Fortner principle were found to be a sensitive and cost-effective alternative that might be used in resource-limited settings. 
Fig. 1. Bacteroides fragilis ATCC 25285 (in the upper half of the nutrient medium) cultivate onto Wilkins-Chalgren anaerobic agar by Fortner principle. Staphylococcus aureus (A) and Escherichia coli (B) are seeded in the lower half of the culture medium. Although the oxygen-consuming microorganisms did not survive and accordingly did not consume the oxygen in the Petri dish, Bacteroides fragilis which is a strict anaerobe, demonstrate growth.
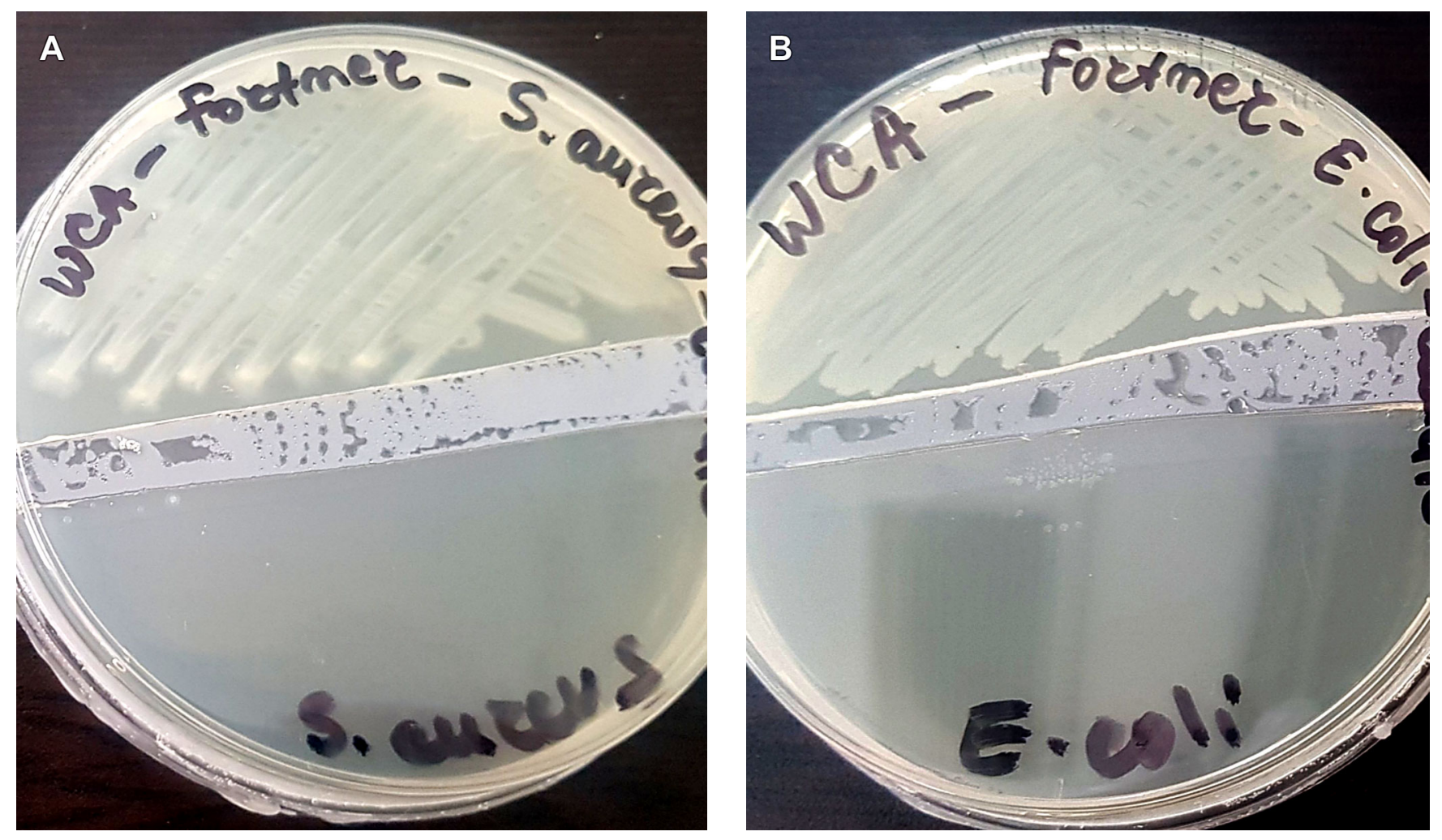

\section{REFERENCES:}

1. Wexler AG, Goodman AL. An insider's perspective: Bacteroides as a window into the microbiome. Nat Microbiol. 2017 Apr 25;2:17026. [PubMed]

2. Itzhak Brook. Bacteroides infections. Medscape. Jun 10, 2019. [Internet]

3. Garrett WS, Onderdonk AB. 248. Bacteroides, Prevotella, Porphyromonas, and Fusobacterium Species (and Other Medically Important Anaerobic GramNegative Bacilli). Section K. Spirochetes. In: Mandell, Douglas, and Bennett's Principles and Practice of Infectious Diseases. Authors: Bennett J, Dolin R, Blaser MJ. 8th Edition. 2-Volume Set. Saunders. 28th August 2014. p. 2773-80. [Internet]

4. Jamal W, Khodakhast FB, AlAzmi A, Soki J, AlHashem G, Rotimi VO. Preva- lence and antimicrobial susceptibility of enterotoxigenic extra-intestinal Bacteroides fragilis among 13-year collection of isolates in Kuwait. BMC Microbiol. 2020 Jan 15;20(1):14. [PubMed]

5. Baughn AD, Malamy MH. The strict anaerobe Bacteroides fragilis grows in and benefits from nanomolar concentrations of oxygen. Nature. 2004 Jan;427(6973):4414. [PubMed]

6. Bacic MK, Smith CJ. Laboratory maintenance and cultivation of bacteroides species. Curr Protoc Microbiol. 2008 May; Chapter 13:Unit 13C.1. [PubMed]

7. Karmali MA, Fleming PC. Application of the Fortner principle to isolation of Campylobacter from stools. J Clin Microbiol.
1979 Aug;10(2):245-247. [PubMed]

8. Popovic-Uroic T, Sterk-Kuzmanovic $\mathrm{N}$. Comparison of two simple microaerobic atmospheres for cultivation of thermophilic campylobacters. J Clin Pathol. 1992 Jan;45(1):87-8. [PubMed]

9. Maiti PK, Haldar J, Mukherjee P, Dey R. Anaerobic culture on growth efficient bi-layered culture plate in a modified candle jar using a rapid and slow combustion system. Indian J Med Microbiol. 2013 Apr-Jun;31(2):173-6. [PubMed]

10. Saha US, Misra R, Tiwari D, Prasad KN. A cost-effective anaerobic culture method \& its comparison with a standard method. Indian J Med Res. 2016 Oct;144(4):611-613. [PubMed]

Please cite this article as: Ermenlieva NM, Georgieva EP, Stamova SY, Georgieva SF, Mihaylova SG. Two alternative methods for anaerobic cultivation of Bacteroides fragilis ATCC 25285 compared to the efficiency of cultivation in a Gas Pack system. J of IMAB. 2021 Oct-Dec;27(4):4035-4037. DOI: https://doi.org/10.5272/jimab.2021274.4035

Received: 08/03/2021; Published online: 13/10/2021

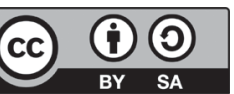

J of IMAB. 2021 Oct-Dec;27(4)
Address for correspondence:

Neli Mitkova Ermenlieva, Department of Microbiology and Virology, Faculty of Medicine, Medical University - Varna; 3, Bregalniza Str., 9002 Varna, Bulgaria

E-mail: n.ermenlieva@abv.bg

https://www.journal-imab-bg.org

4037 